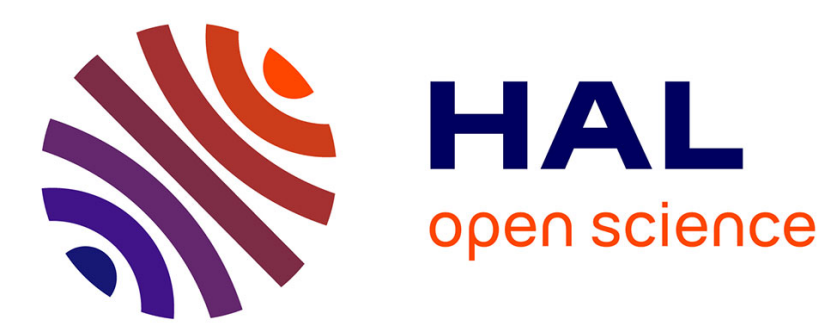

\title{
MATHEMATICAL ANALYSIS OF A PARABOLIC-ELLIPTIC MODEL FOR BRAIN LACTATE KINETICS
}

Alain Miranville

\section{- To cite this version:}

Alain Miranville. MATHEMATICAL ANALYSIS OF A PARABOLIC-ELLIPTIC MODEL FOR BRAIN LACTATE KINETICS. 2017. hal-01445518

\section{HAL Id: hal-01445518 \\ https://hal.science/hal-01445518}

Preprint submitted on 25 Jan 2017

HAL is a multi-disciplinary open access archive for the deposit and dissemination of scientific research documents, whether they are published or not. The documents may come from teaching and research institutions in France or abroad, or from public or private research centers.
L'archive ouverte pluridisciplinaire HAL, est destinée au dépôt et à la diffusion de documents scientifiques de niveau recherche, publiés ou non, émanant des établissements d'enseignement et de recherche français ou étrangers, des laboratoires publics ou privés. 


\title{
MATHEMATICAL ANALYSIS OF A PARABOLIC-ELLIPTIC MODEL FOR BRAIN LACTATE KINETICS
}

\author{
ALAIN MIRANVILLE
}

\begin{abstract}
Our aim in this paper is to study properties of a parabolic-elliptic system related with brain lactate kinetics. These equations are obtained from a reaction-diffusion system, when a small parameter vanishes. In particular, we prove the existence and uniqueness of nonnegative solutions and obtain error estimates on the difference of the solutions to the initial reaction-diffusion system and those to the limit one, on bounded time intervals. We also study the linear stability of the unique spatially homogeneous equilibrium.
\end{abstract}

\section{INTRODUCTION}

The following system of ODE's:

$$
\begin{gathered}
\frac{d u}{d t}+\kappa\left(\frac{u}{k+u}-\frac{v}{k^{\prime}+v}\right)=J, \kappa, k, k^{\prime}, J>0, \\
\varepsilon \frac{d v}{d t}+F v+\kappa\left(\frac{v}{k^{\prime}+v}-\frac{u}{k+u}\right)=F L, \varepsilon, F, L>0,
\end{gathered}
$$

where $\varepsilon$ is a small parameter, was proposed and studied as a model for brain lactate kinetics (see [5], [8], [9] and [10]; see also [4]). In this context, $u=u(t)$ and $v=v(t)$ correspond to the lactate concentrations in an interstitial (i.e., extra-cellular) domain and in a capillary domain, respectively. Furthermore, the nonlinear term $\kappa\left(\frac{u}{k+u}-\frac{v}{k^{\prime}+v}\right)$ stands for a co-transport through the brain-blood boundary (see [11]). Finally, $J$ and $F$ are forcing and input terms, respectively, assumed frozen (more generally, $J$ depends on $t$ and $u$ and accounts for the interactions with a third intracellular compartment (which includes both neurons and astrocytes), while $F=F(t)$ (an applied electrical stimulus; see [7]) is piecewise linear and periodic). This model has essential applications to the therapeutic management of glioma (also called glial tumors); see [8] for thorough discussions on this issue.

Let us assume that $u(0)$ and $v(0)$ are nonnegative (recall that $u$ and $v$ are concentrations and are thus expected to be nonnegative). Then, noting that, if $u(0)=0$, then $\frac{d u}{d t}(0)>0$ and, if $v(0)=0$, then $\frac{d v}{d t}(0)>0$, it follows from Cauchy-Lipschitz theorem that, for $t>0$ small, $u$ and $v$ exist and are nonnegative. This also yields that the solutions are defined and remain nonnegative on the whole interval $\mathbb{R}^{+}$; indeed, it is not difficult to prove that

2010 Mathematics Subject Classification. 35M33, 35K57, 35K67, 35B45.

Key words and phrases. Brain lactate kinetics, parabolic-elliptic system, reaction-diffusion system, limit system, nonnegative solutions, well-posedness, error estimates, linear stability. 
they are bounded on finite time intervals. Furthermore, in [5], [8], [9] and [10], questions related to the stability of the unique equilibrium were addressed. This constitutes an essential point in the modeling, since, as discussed in [8], a therapeutic perspective of such a result is to have the steady state outside the viability domain, where cell necrosis occurs. Finally, in [9], justifications for the dip and buffering which are observed in experiments (see [7]) were given, based on geometrical arguments and averaging theory on a slow manifold.

We can note that the above ODE's model does not account for spatial diffusion. Taking this into account would be relevant and desirable from a biological point of view. The simplest possible corresponding PDE's (reaction-diffusion) system, accounting for spatial diffusion, reads

$$
\begin{gathered}
\frac{\partial u}{\partial t}-\alpha \Delta u+\kappa\left(\frac{u}{k+u}-\frac{v}{k^{\prime}+v}\right)=J, \alpha>0, \\
\varepsilon \frac{\partial v}{\partial t}-\beta \Delta v+F v+\kappa\left(\frac{v}{k^{\prime}+v}-\frac{u}{k+u}\right)=F L, \beta>0,
\end{gathered}
$$

where $u=u(x, t)$ and $v=v(x, t)$, which we consider in a bounded and regular domain $\Omega$ of $\mathbb{R}^{N}, N=1,2$ or 3 , together with Neumann boundary conditions,

$$
\frac{\partial u}{\partial \nu}=\frac{\partial v}{\partial \nu}=0 \text { on } \Gamma
$$

where $\Gamma=\partial \Omega$ and $\nu$ is the unit outer normal vector. Note that the terms $-\alpha \Delta u$ and $-\beta \Delta v$ correspond to random motions. Note however that more precise models should account for the geometry, i.e., the different compartments (interstitial, capillary), so that (1.3)-(1.4) should be viewed as a very first step towards PDE's models for brain lactate kinetics. We will consider more realistic models elsewhere.

We studied in [6] the existence, uniqueness and regularity of nonnegative solutions to (1.3)-(1.4) (note that the mathematical analysis of (1.3)-(1.4) (and, in particular, the wellposedness) appears to be challenging, due to the coupling terms, especially for negative initial data (though biologically irrelevant, this makes sense from a mathematical point of view); this is also the case for the ODE's model (1.1)-(1.2)). We further established the linear (exponential) stability of the unique spatially homogeneous equilibrium. We also mention [12] in which we proved the existence, uniqueness and regularity of the solutions to the following singular reaction-diffusion equation:

$$
\frac{\partial u}{\partial t}-\Delta u+F u+\kappa \frac{u}{k+u}=f(x, t), F \geq 0
$$

corresponding to the case where either $u$ or $v$ is known in (1.3) and (1.4); we can also think of (1.5) as an equation in each compartment, assuming that the lactate concentration is known in the other one.

Our aim in this paper is to study the limit system 


$$
\begin{gathered}
\frac{\partial u}{\partial t}-\alpha \Delta u+\kappa\left(\frac{u}{k+u}-\frac{v}{k^{\prime}+v}\right)=J, \\
-\beta \Delta v+F v+\kappa\left(\frac{v}{k^{\prime}+v}-\frac{u}{k+u}\right)=F L,
\end{gathered}
$$

corresponding to $\varepsilon=0$ in (1.4). We prove the existence and uniqueness of nonnegative solutions to (1.6)-(1.7). We then prove that the solutions to the initial reaction-diffusion system converge to those to the limit parabolic-elliptic one, on finite time intervals, and provide an error estimate in terms of $\varepsilon$. We finally study the linear stability of the unique spatially homogeneous equilibrium. We can note that a similar analysis would also be relevant in the context of the ODE's model (1.1)-(1.2). Though some of the results obtained here could apply (in a simpler way) to this system, this will be considered in more details elsewhere.

Notation: We denote by $((\cdot, \cdot))$ the usual $L^{2}$-scalar product, with associated norm $\|\cdot\|$. More generally, $\|\cdot\|_{X}$ denotes the norm on the Banach space $X$ and, if $X$ is a Hilbert space, $((\cdot, \cdot))_{X}$ denotes the associated scalar product.

Throughout the paper, the same letters $c, c^{\prime}$ and $c^{\prime \prime}$ denote positive constants which may vary from line to line. Similarly, the same letter $Q$ denotes continuous and monotone increasing (with respect to each argument) functions which may vary from line to line.

\section{The CASE $\varepsilon>0$}

We consider the following initial and boundary value problem:

$$
\begin{gathered}
\frac{\partial u}{\partial t}-\alpha \Delta u+\kappa\left(\frac{u}{k+u}-\frac{v}{k^{\prime}+v}\right)=J \\
\varepsilon \frac{\partial v}{\partial t}-\beta \Delta v+F v+\kappa\left(\frac{v}{k^{\prime}+v}-\frac{u}{k+u}\right)=F L, \varepsilon>0 \\
\frac{\partial u}{\partial \nu}=\frac{\partial v}{\partial \nu}=0 \text { on } \Gamma \\
\left.u\right|_{t=0}=u_{0},\left.v\right|_{t=0}=v_{0} .
\end{gathered}
$$

Note that (2.1)-(2.2) are equivalent to

$$
\begin{gathered}
\frac{\partial u}{\partial t}-\alpha \Delta u+\kappa\left(\frac{k^{\prime}}{k^{\prime}+v}-\frac{k}{k+u}\right)=J \\
\varepsilon \frac{\partial v}{\partial t}-\beta \Delta v+F v+\kappa\left(\frac{k}{k+u}-\frac{k^{\prime}}{k^{\prime}+v}\right)=F L .
\end{gathered}
$$

We assume that 


$$
\left(u_{0}, v_{0}\right) \in H_{\mathrm{N}}^{2}(\Omega)^{2}, u_{0} \geq 0, v_{0} \geq 0 \text { a.e. } x,
$$

where

$$
H_{\mathrm{N}}^{2}(\Omega)=\left\{w \in H^{2}(\Omega), \frac{\partial w}{\partial \nu}=0 \text { on } \Gamma\right\} .
$$

We proved in [6] the

Theorem 2.1. We assume that (2.7) holds. Then, (2.1)-(2.4) possesses a unique strong solution $(u, v)$ such that

$$
u \geq 0, v \geq 0 \text { a.e. }(x, t)
$$

and, $\forall T>0$,

$$
\begin{gathered}
(u, v) \in L^{\infty}\left(0, T ; H_{\mathrm{N}}^{2}(\Omega)^{2}\right) \cap L^{2}\left(0, T ; H^{3}(\Omega)^{2}\right), \\
\left(\frac{\partial u}{\partial t}, \frac{\partial v}{\partial t}\right) \in L^{\infty}\left(0, T ; L^{2}(\Omega)^{2}\right) \cap L^{2}\left(0, T ; H^{1}(\Omega)^{2}\right) .
\end{gathered}
$$

Furthermore,

$$
\|u(t)\|_{L^{\infty}(\Omega)} \leq\left\|u_{0}\right\|_{L^{\infty}(\Omega)}+(J+\kappa) t, t \geq 0,
$$

and

$$
\|v(t)\|_{L^{\infty}(\Omega)} \leq e^{-\frac{F}{\varepsilon} t}\left\|v_{0}\right\|_{L^{\infty}(\Omega)}+\frac{F L+\kappa}{F}, t \geq 0 .
$$

Finally, if $M \geq \frac{F L+\kappa}{F}$ and $0 \leq v_{0} \leq M$ a.e. $x$, then $0 \leq v \leq M$ a.e. $(x, t)$.

Remark 2.2. As far as the above regularity is concerned, the corresponding constants in [6] depend on $\varepsilon$, i.e., they are not bounded uniformly with respect to $\varepsilon$ as this quantity goes to 0 . However, it is not difficult, reading the details, to see that most constants can be made independent of $\varepsilon$, yielding regularity estimates on $u, \frac{\partial u}{\partial t}$ and $v$ which are uniform with respect to $\varepsilon$ as $\varepsilon \rightarrow 0$. Now, we have not been able to derive, at least in a straightforward way, such uniform estimates on $\frac{\partial v}{\partial t}$ which would allow us to pass to the limit in (2.2) (say, in a weak (variational) form) to deduce the existence of a solution to the limit problem corresponding to $\varepsilon=0$ (see however Section 4). We will thus give a direct proof of existence for the limit problem which also has an interest on its own.

Remark 2.3. (i) It follows from the above that the capillary lactate concentration is uniformly (with respect to time) bounded. However, we have not been able to derive a similar upper bound on the interstitial lactate concentration $u$. We can note that, in the biological model, outside a bounded viability domain, cell necrosis occurs (see [8]), meaning that one expects viable trajectories to be uniformly bounded.

(ii) Multiplying (2.1) by $u+k$, integrating over $\Omega$ and by parts, we obtain 
where

$$
\frac{d E}{d t}+\alpha\|\nabla u\|^{2}+\kappa\|u\|_{L^{1}(\Omega)} \leq\left(\left(J+\frac{\kappa v}{k^{\prime}+v}, u+k\right)\right)
$$

$$
E=\frac{1}{2} u^{2}+k u
$$

Noting that $v$ is uniformly bounded (we assume that, say, $0 \leq v_{0} \leq \frac{F L+\kappa}{F}$ ), we take, for $\kappa, J, F$ and $L$ given, $J$ small enough and $k^{\prime}$ large enough such that

We thus deduce that

$$
J+\frac{\kappa v}{k^{\prime}+v}<\kappa
$$

$$
\frac{d E}{d t}+\alpha\|\nabla u\|^{2}+c\|u\|_{L^{1}(\Omega)} \leq c^{\prime}, c>0
$$

which yields, noting that

$$
\begin{gathered}
\alpha\|\nabla u\|^{2}+c\|u\|_{L^{1}(\Omega)} \geq c^{\prime}\left(\|\nabla u\|+\|u\|_{L^{1}(\Omega)}\right)-c^{\prime \prime} \\
\geq c^{\prime}\|u\|-c^{\prime \prime},
\end{gathered}
$$

the differential inequality

$$
\frac{d E}{d t}+c \sqrt{E} \leq c^{\prime}, c>0
$$

Set $E^{*}=\left(\frac{c^{\prime}}{c}\right)^{2}$, where $c$ and $c^{\prime}$ are the same constants as in (2.9), so that

$$
\frac{d E^{*}}{d t}+c \sqrt{E^{*}}=c^{\prime}
$$

It then follows from comparison arguments that

$$
E(t) \leq \max \left(E(0), E^{*}\right), t \geq 0,
$$

and we finally deduce that the $L^{2}$-norm of $u$ is uniformly bounded.

\section{THE CASE $\varepsilon=0$}

We consider in this section the following initial and boundary value problem:

$$
\begin{gathered}
\frac{\partial u}{\partial t}-\alpha \Delta u+\kappa\left(\frac{k^{\prime}}{k^{\prime}+v}-\frac{k}{k+u}\right)=J, \\
-\beta \Delta v+F v+\kappa\left(\frac{k}{k+u}-\frac{k^{\prime}}{k^{\prime}+v}\right)=F L, \\
\frac{\partial u}{\partial \nu}=\frac{\partial v}{\partial \nu}=0 \text { on } \Gamma,
\end{gathered}
$$




$$
\left.u\right|_{t=0}=u_{0}
$$

We assume that

$$
u_{0} \in H_{\mathrm{N}}^{2}(\Omega), u_{0} \geq 0 \text { a.e. } x \text {. }
$$

Remark 3.1. It follows from (3.2) that

$$
-\beta \Delta v(0)+F v(0)-\frac{k^{\prime}}{k^{\prime}+v(0)}=F L-\frac{k}{k+u_{0}} .
$$

We will see below that this allows to define in a unique way $v(0)$ such that $v(0) \geq 0$ a.e. $x$.

3.1. Existence and uniqueness of solutions to an auxiliary problem. We consider the following modified initial and boundary value problem:

$$
\begin{gathered}
\frac{\partial u}{\partial t}-\alpha \Delta u+\kappa\left(\frac{u}{k+|u|}-\frac{v}{k^{\prime}+|v|}\right)=J, \\
-\beta \Delta v+F v+\kappa\left(\frac{v}{k^{\prime}+|v|}-\frac{u}{k+|u|}\right)=F L, \\
\frac{\partial u}{\partial \nu}=\frac{\partial v}{\partial \nu}=0 \text { on } \Gamma, \\
\left.u\right|_{t=0}=u_{0} .
\end{gathered}
$$

We associate with (3.6)-(3.9) the following weak/variational formulation, for $T>0$ given:

Find $(u, v):[0, T] \rightarrow H^{1}(\Omega)^{2}$ such that

$$
\frac{d}{d t}((u, \phi))+\alpha((\nabla u, \nabla \phi))+\left(\left(\varphi_{k}(u), \phi\right)\right)-\left(\left(\varphi_{k^{\prime}}(v), \phi\right)\right)=((J, \phi)), \forall \phi \in H^{1}(\Omega),
$$

$(3.11) \beta((\nabla v, \nabla \psi))+F((v, \psi))+\left(\left(\varphi_{k^{\prime}}(v), \psi\right)\right)-\left(\left(\varphi_{k}(u), \psi\right)\right)=((F L, \psi)), \forall \psi \in H^{1}(\Omega)$, in the sense of distributions, and

$$
u(0)=u_{0} \text { in } L^{2}(\Omega),
$$

where we have set, for $c>0$ given,

$$
\varphi_{c}(s)=\frac{\kappa s}{c+|s|}, s \in \mathbb{R} .
$$

We can note that $\varphi_{c}$ is bounded (with $\left.\left|\varphi_{c}\right| \leq \kappa\right)$ and of class $\mathcal{C}^{1}$, with $\varphi_{c}^{\prime}(s)=\frac{\kappa c}{(c+|s|)^{2}}$, so that $\varphi_{c}$ is also Lipschitz continuous, with Lipschitz constant $\frac{\kappa}{c}$. 
Let then $0=\lambda_{1}<\lambda_{2} \leq \cdots$ be the eigenvalues of the minus Laplace operator associated with Neumann boundary conditions and $w_{1}, w_{2}, \cdots$ be associated eigenvectors such that the $w_{j}$ 's form an orthonormal in $L^{2}(\Omega)$ and orthogonal in $H^{1}(\Omega)$ basis. Setting

$$
V_{m}=\operatorname{Span}\left(w_{1}, \cdots, w_{m}\right), m \in \mathbb{N},
$$

we consider the following approximated problem, for $T>0$ given:

Find $\left(u_{m}, v_{m}\right):[0, T] \rightarrow V_{m} \times V_{m}$ such that

$$
\begin{aligned}
& \frac{d}{d t}\left(\left(u_{m}, \phi\right)\right)+\alpha\left(\left(\nabla u_{m}, \nabla \phi\right)\right)+\left(\left(\varphi_{k}\left(u_{m}\right), \phi\right)\right)-\left(\left(\varphi_{k^{\prime}}\left(v_{m}\right), \phi\right)\right)=((J, \phi)), \forall \phi \in V_{m}, \\
& \beta\left(\left(\nabla v_{m}, \nabla \psi\right)\right)+F\left(\left(v_{m}, \psi\right)\right)+\left(\left(\varphi_{k^{\prime}}\left(v_{m}\right), \psi\right)\right)-\left(\left(\varphi_{k}\left(u_{m}\right), \psi\right)\right)=((F L, \psi)), \forall \psi \in V_{m},
\end{aligned}
$$

in the sense of distributions, and

$$
u_{m}(0)=u_{0 m}
$$

where $u_{0 m}=P_{m} u_{0}, P_{m}$ being the orthogonal projector (for the $L^{2}$-norm) from $L^{2}(\Omega)$ onto $V_{m}$.

For $w \in V_{m}$ given, we consider the following elliptic problem:

Find $z \in V_{m}$ such that

$$
a(z, \phi)+\left(\left(\varphi_{k^{\prime}}(z), \phi\right)\right)=\left(\left(F L+\varphi_{k}(w), \phi\right)\right), \forall \phi \in V_{m}
$$

where

$$
a(\cdot, \cdot)=\beta((\nabla \cdot, \nabla \cdot))+F((\cdot, \cdot))
$$

is bilinear, symmetric, continuous and coercive on $V_{m}$ (and also on $H^{1}(\Omega)$ ). Let then $R=R_{m}$ be the operator defined by

$$
R: V_{m} \rightarrow V_{m}, z \mapsto R(z),
$$

where

$$
((R(z), \phi))_{H^{1}(\Omega)}=a(z, \phi)+\left(\left(\varphi_{k^{\prime}}(z), \phi\right)\right)-\left(\left(F L+\varphi_{k}(w), \phi\right)\right), \forall \phi \in V_{m} .
$$

It is clear that this operator is well defined and continuous (since $\varphi_{k^{\prime}}$ is Lipschitz continuous). Furthermore, there holds, for $z \in V_{m}$,

$$
\begin{aligned}
((R(z), z))_{H^{1}(\Omega)} & =a(z, z)+\left(\left(\varphi_{k^{\prime}}(z), z\right)\right)-\left(\left(F L+\varphi_{k}(w), z\right)\right) \\
& \geq c\|z\|_{H^{1}(\Omega)}^{2}-c^{\prime}\|z\|, c>0
\end{aligned}
$$

(note indeed that $w$ is given and recall that $\varphi_{k}$ and $\varphi_{k^{\prime}}$ are bounded). Therefore,

so that

$$
((R(z), z))_{H^{1}(\Omega)} \geq c\|z\|_{H^{1}(\Omega)}^{2}-c^{\prime}
$$




$$
((R(z), z))_{H^{1}(\Omega)} \geq 0 \text { whenever }\|z\|_{H^{1}(\Omega)} \geq \sqrt{\frac{c^{\prime}}{c}} .
$$

It thus follows from the Brouwer fixed point theorem that there exists $z \in V_{m},\|z\|_{H^{1}(\Omega)} \leq$ $\sqrt{\frac{c^{\prime}}{c}}$, such that

$$
R(z)=0 \text { in } V_{m}
$$

(see, e.g., [14]), which is equivalent to (3.16). Note that all constants here (and also below) are independent of $m$. This thus defines a mapping $\mathcal{F}=\mathcal{F}_{m}$,

$$
\mathcal{F}: V_{m} \rightarrow V_{m}, w \mapsto z=\mathcal{F}(w) .
$$

Let then $\left(w_{1}, w_{2}\right) \in V_{m} \times V_{m}$ and set $z_{i}=\mathcal{F}\left(w_{i}\right), i=1,2$. We have, setting $z=z_{1}-z_{2}$ and $w=w_{1}-w_{2}$,

$$
a(z, \phi)+\left(\left(\varphi_{k^{\prime}}\left(z_{1}\right)-\varphi_{k^{\prime}}\left(z_{2}\right), \phi\right)\right)=\left(\left(\varphi_{k}\left(w_{1}\right)-\varphi_{k}\left(w_{2}\right), \phi\right)\right), \forall \phi \in V_{m} .
$$

Taking $\phi=z$ and noting that $\varphi_{k^{\prime}}$ is monotone increasing and $\varphi_{k}$ is Lipschitz continuous, we obtain

whence

$$
\|z\|_{H^{1}(\Omega)}^{2} \leq c\|w\|\|z\|
$$

$$
\left\|\mathcal{F}\left(w_{1}\right)-\mathcal{F}\left(w_{2}\right)\right\|_{H^{1}(\Omega)} \leq c\left\|w_{1}-w_{2}\right\|,
$$

which yields that $\mathcal{F}$ is Lipschitz continuous on $V_{m}$ (both for the $L^{2}$ and $H^{1}$-norms); this also yields that $\mathcal{F}$ is indeed a mapping, since $w_{1}=w_{2}$ implies $z_{1}=z_{2}$.

It follows from the above that (3.13)-(3.15) is equivalent to

Find $u_{m}:[0, T] \rightarrow V_{m}$ such that

$$
\begin{aligned}
\frac{d}{d t}\left(\left(u_{m}, \phi\right)\right)+\alpha\left(\left(\nabla u_{m},\right.\right. & \nabla \phi))+\left(\left(\varphi_{k}\left(u_{m}\right), \phi\right)\right)-\left(\left(\varphi_{k^{\prime}} \circ \mathcal{F}\left(u_{m}\right), \phi\right)\right) \\
= & ((J, \phi)), \forall \phi \in V_{m},
\end{aligned}
$$

in the sense of distributions,

$$
u_{m}(0)=u_{0 m}
$$

and then set $v_{m}=\mathcal{F}\left(u_{m}\right)$.

Since $\varphi_{k}$ and $\varphi_{k^{\prime}}$ are Lipschitz continuous on $\mathbb{R}$ and $\mathcal{F}$ is Lipschitz continuous on $V_{m}$ with respect to the $L^{2}$-norm, it is easy to prove that (3.19)-(3.20) possesses a (unique) solution $u_{m} \in L^{\infty}\left(0, T ; L^{2}(\Omega)\right) \cap L^{2}\left(0, T ; H^{1}(\Omega)\right)$ (see, e.g., [13]), whence, setting $v_{m}=\mathcal{F}\left(u_{m}\right)$, the existence of a solution $\left(u_{m}, v_{m}\right)$ to $(3.13)$-(3.15) such that $v_{m} \in L^{\infty}\left(0, T ; H^{1}(\Omega)\right)$. We also note that $\frac{\partial u_{m}}{\partial t} \in L^{2}\left(0, T ; H^{-1}(\Omega)\right)$, so that $u_{m} \in \mathcal{C}\left([0, T] ; L^{2}(\Omega)\right)$.

Writing $u_{m}(t)=\sum_{i=1}^{m} d_{i, m}(t) w_{i}$, taking $\phi=\lambda_{i} w_{i}$ in (3.19), multiplying the resulting equality by $d_{i, m}$ and summing over $i$, we obtain 


$$
\frac{1}{2} \frac{d}{d t}\left\|\nabla u_{m}\right\|^{2}+\alpha\left\|\Delta u_{m}\right\|^{2}-\left(\left(\varphi_{k}\left(u_{m}\right), \Delta u_{m}\right)\right)+\left(\left(\varphi_{k^{\prime}} \circ \mathcal{F}\left(u_{m}\right), \Delta u_{m}\right)\right)=0,
$$

which yields, recalling that $\varphi_{k}$ and $\varphi_{k^{\prime}}$ are bounded,

$$
\frac{d}{d t}\left\|\nabla u_{m}\right\|^{2}+\alpha\left\|\Delta u_{m}\right\|^{2} \leq c
$$

whence estimates on $u_{m}$ in $L^{\infty}\left(0, T ; H^{1}(\Omega)\right)$ and $L^{2}\left(0, T ; H^{2}(\Omega)\right)$. It thus follows that $\frac{\partial u_{m}}{\partial t} \in L^{\infty}\left(0, T ; H^{-1}(\Omega)\right) \cap L^{2}\left(0, T ; L^{2}(\Omega)\right)$.

Since the above regularity estimates are uniform with respect to $m$, we deduce from classical Aubin-Lions compactness theorems that, at least for a subsequence which we do not relabel,

$$
\begin{gathered}
u_{m} \rightarrow u \text { in } L^{\infty}\left(0, T ; H^{1}(\Omega)\right) \text { weak star, in } L^{2}\left(0, T ; H^{2}(\Omega)\right) \text { weak, } \\
\text { in } \mathcal{C}\left([0, T] ; L^{2}(\Omega)\right) \text { and a.e. }(x, t) \in \Omega \times(0, T), \\
v_{m} \rightarrow v \text { in } L^{\infty}\left(0, T ; H^{1}(\Omega)\right) \text { weak star, }
\end{gathered}
$$

for some functions $u$ and $v$. Actually, since $v_{m}=\mathcal{F}\left(u_{m}\right)$ and $\mathcal{F}$ is Lipschitz continuous with respect to the $L^{2}$-norm, we can see that $\left(v_{m}\right)$ is a Cauchy sequence in $\mathcal{C}\left([0, T] ; L^{2}(\Omega)\right.$ ) (note indeed that, if $m^{\prime} \geq m$, then $V_{m} \subset V_{m^{\prime}}$ and that the constant $c$ in (3.18) is independent of $m$ ), so that

$$
v_{m} \rightarrow v \text { in } \mathcal{C}\left([0, T] ; L^{2}(\Omega)\right) .
$$

Recalling finally that $\varphi_{k}$ and $\varphi_{k^{\prime}}$ are Lipschitz continuous, this is sufficient to pass to the limit in (3.13)-(3.15) and deduce the existence of a solution $(u, v)$ to (3.10)-(3.12) (note that the initial condition $u_{0}=u(0)$ makes sense; actually, $v(0)$ also makes sense). Indeed, we need to pass to the limit in relations of the form

$$
\begin{gathered}
\int_{0}^{T}\left[-\left(\left(u_{m}, \phi\right)\right) \theta^{\prime}(t)+\alpha\left(\left(\nabla u_{m}, \nabla \phi\right)\right) \theta(t)+\left(\varphi_{k}\left(u_{m}\right), \phi\right)\right) \theta(t) \\
\left.-\left(\left(\varphi_{k^{\prime}}\left(v_{m}\right), \phi\right)\right) \theta(t)-((J, \phi)) \theta(t)\right] d t=0
\end{gathered}
$$

and

$\int_{0}^{T}\left[\beta\left(\left(\nabla v_{m}, \nabla \psi\right)\right)+F\left(\left(v_{m}, \psi\right)\right)+\left(\left(\varphi_{k^{\prime}}\left(v_{m}\right), \psi\right)\right)-\left(\left(\varphi_{k}\left(u_{m}\right), \psi\right)\right)-((F L, \psi))\right] \theta(t) d t=0$, for $(\phi, \psi) \in H^{1}(\Omega)^{2}$ and $\theta \in \mathcal{D}(0, T)$. More precisely, we have the

Theorem 3.2. We assume that $u_{0} \in H^{1}(\Omega)$. Then, (3.10)-(3.12) possesses a unique solution $(u, v)$ such that, $\forall T>0$,

$$
\begin{gathered}
u \in L^{\infty}\left(0, T ; H^{1}(\Omega)\right) \cap L^{2}\left(0, T ; H^{2}(\Omega)\right) \cap \mathcal{C}\left([0, T] ; L^{2}(\Omega)\right), \\
v \in L^{\infty}\left(0, T ; H^{1}(\Omega)\right) \cap \mathcal{C}\left([0, T] ; L^{2}(\Omega)\right)
\end{gathered}
$$

and 


$$
\frac{\partial u}{\partial t} \in L^{\infty}\left(0, T ; H^{-1}(\Omega)\right) \cap L^{2}\left(0, T ; L^{2}(\Omega)\right) .
$$

Proof. There remains to prove the uniqueness.

Let thus $\left(u_{1}, v_{1}\right)$ and $\left(u_{2}, v_{2}\right)$ be two such solutions, with initial data $u_{0,1}$ and $u_{0,2}$, respectively. We have, setting $(u, v)=\left(u_{1}-u_{2}, v_{1}-v_{2}\right)$ and $u_{0}=u_{0,1}-u_{0,2}$,

$$
\begin{aligned}
\frac{d}{d t}((u, \phi))+\alpha((\nabla u, \nabla \phi)) & +\left(\left(\varphi_{k}\left(u_{1}\right)-\varphi_{k}\left(u_{2}\right), \phi\right)\right)-\left(\left(\varphi_{k^{\prime}}\left(v_{1}\right)-\varphi_{k^{\prime}}\left(v_{2}\right), \phi\right)\right) \\
& =0, \forall \phi \in H^{1}(\Omega),
\end{aligned}
$$

$$
\begin{gathered}
\beta((\nabla v, \nabla \psi))+F((v, \psi))+\left(\left(\varphi_{k^{\prime}}\left(v_{1}\right)-\varphi_{k^{\prime}}\left(v_{2}\right), \psi\right)\right)-\left(\left(\varphi_{k}\left(u_{1}\right)-\varphi_{k}\left(u_{2}\right), \psi\right)\right) \\
=0, \forall \psi \in H^{1}(\Omega), \\
u(0)=u_{0} .
\end{gathered}
$$

Taking $\phi=u$ and $\psi=v$, we obtain, recalling that $\varphi_{k}$ and $\varphi_{k^{\prime}}$ are monotone increasing and Lipschitz continuous,

$$
\frac{1}{2} \frac{d}{d t}\|u\|^{2}+\alpha\|\nabla u\|^{2} \leq c\|u\|\|v\|
$$

and

$$
\beta\|\nabla v\|^{2}+F\|v\|^{2} \leq c\|u\|\|v\|,
$$

respectively. In particular, it follows from (3.25) that

$$
\|v\| \leq c\|u\|
$$

which, injected into (3.24), yields

$$
\frac{d}{d t}\|u\|^{2} \leq c\|u\|^{2}
$$

whence, owing to Gronwall's lemma,

$$
\|u(t)\| \leq e^{c t}\left\|u_{0}\right\|, t \geq 0 .
$$

We deduce from (3.26) and (3.28) the uniqueness, as well as the continuous dependence with respect to the initial data in the $L^{2}$-norm. 
3.2. Existence and uniqueness of nonnegative solutions. We first prove additional regularity results on the solutions to (3.10)-(3.11), assuming that (3.5) holds. This can be fully justified within the Galerkin scheme considered above.

Taking $\psi=-\Delta v$ in (3.11), we have

$$
\beta\|\Delta v\|^{2}+F\|\nabla v\|^{2}=\left(\left(\varphi_{k^{\prime}}(v), \Delta v\right)\right)-\left(\left(\varphi_{k}(u), \Delta v\right)\right),
$$

which yields, recalling that $\varphi_{k}$ and $\varphi_{k^{\prime}}$ are bounded,

$$
\frac{\beta}{2}\|\Delta v\|^{2}+F\|\nabla v\|^{2} \leq c
$$

whence estimates on $v$ in $L^{\infty}\left(0, T ; H^{2}(\Omega)\right), \forall T>0$.

Taking then $\phi=\Delta^{2} u$ in (3.10) and $\psi=\Delta^{2} v$ in (3.11), we obtain

and

$$
\frac{1}{2} \frac{d}{d t}\|\Delta u\|^{2}+\alpha\|\nabla \Delta u\|^{2}=-\left(\left(\varphi_{k}(u), \Delta^{2} u\right)\right)+\left(\left(\varphi_{k^{\prime}}(v), \Delta^{2} u\right)\right)
$$

$$
\beta\|\nabla \Delta v\|^{2}+F\|\Delta v\|^{2}=-\left(\left(\varphi_{k^{\prime}}(v), \Delta^{2} v\right)\right)+\left(\left(\varphi_{k}(u), \Delta^{2} v\right)\right),
$$

respectively. Noting that

$$
\left|\left(\left(\varphi_{k}(u), \Delta^{2} u\right)\right)\right|=\left|\left(\left(\varphi_{k}^{\prime}(u) \nabla u, \nabla \Delta u\right)\right)\right| \leq c\|\nabla u\|\|\nabla \Delta u\|,
$$

we find, proceeding in a similar way for the other terms,

$$
\frac{d}{d t}\|\Delta u\|^{2}+\alpha\|\nabla \Delta u\|^{2} \leq c\left(\|\nabla u\|^{2}+\|\nabla v\|^{2}\right)
$$

and

$$
\frac{\beta}{2}\|\nabla \Delta v\|^{2}+F\|\Delta v\|^{2} \leq c\left(\|\nabla u\|^{2}+\|\nabla v\|^{2}\right),
$$

whence estimates on $u$ and $v$ in $L^{\infty}\left(0, T ; H^{2}(\Omega)\right) \cap L^{2}\left(0, T ; H^{3}(\Omega)\right)$ and $L^{\infty}\left(0, T ; H^{3}(\Omega)\right)$, respectively, $\forall T>0$.

Remark 3.3. This yields that $\frac{\partial u}{\partial t} \in L^{\infty}\left(0, T ; L^{2}(\Omega)\right) \cap L^{2}\left(0, T ; H^{1}(\Omega)\right), \forall T>0$. We further note that the solution to (3.10)-(3.12) is strong, i.e., (3.6)-(3.9) are satisfied almost everywhere.

We can now prove the

Theorem 3.4. We assume that (3.5) holds. Then, (3.1)-(3.4) possesses a unique strong solution $(u, v)$ such that $u \geq 0, v \geq 0$ a.e. $(x, t)$ and, $\forall T>0$,

$$
\begin{gathered}
u \in L^{\infty}\left(0, T ; H_{\mathrm{N}}^{2}(\Omega)\right) \cap L^{2}\left(0, T ; H^{3}(\Omega)\right), \\
v \in L^{\infty}\left(0, T ; H^{3}(\Omega) \cap H_{\mathrm{N}}^{2}(\Omega)\right) \cap \mathcal{C}\left([0, T] ; L^{2}(\Omega)\right)
\end{gathered}
$$

and 


$$
\frac{\partial u}{\partial t} \in L^{\infty}\left(0, T ; L^{2}(\Omega)\right) \cap L^{2}\left(0, T ; H^{1}(\Omega)\right) .
$$

Proof. Let $(u, v)$ be the unique strong solution to (3.6)-(3.9). Multiplying (3.6) by $-u^{-}$ and (3.7) by $-v^{-}$, where $x^{-}=\max (0,-x)$, we have

$$
\frac{1}{2} \frac{d}{d t}\left\|u^{-}\right\|^{2}+\alpha\left\|\nabla u^{-}\right\|^{2}+\kappa \int_{\Omega} \frac{\left|u^{-}\right|^{2}}{k+|u|} d x+\kappa \int_{\Omega} \frac{v u^{-}}{k^{\prime}+|v|} d x \leq 0
$$

and

$$
\beta\left\|\nabla u^{-}\right\|^{2}+F\left\|u^{-}\right\|^{2}+\kappa \int_{\Omega} \frac{\left|v^{-}\right|^{2}}{k^{\prime}+|v|} d x+\kappa \int_{\Omega} \frac{u v^{-}}{k+|u|} d x \leq 0,
$$

respectively. Writing $v=v^{+}-v^{-}$, where $x^{+}=\max (0, x)$, we deduce from (3.29) that

whence

$$
\frac{1}{2} \frac{d}{d t}\left\|u^{-}\right\|^{2} \leq \kappa \int_{\Omega} \frac{u^{-} v^{-}}{k^{\prime}+|v|} d x
$$

$$
\frac{d}{d t}\left\|u^{-}\right\|^{2} \leq c\left\|u^{-}\right\|\left\|v^{-}\right\|
$$

Proceeding in a similar way for (3.30), we find

whence

$$
F\left\|v^{-}\right\|^{2} \leq c\left\|u^{-}\right\|\left\|v^{-}\right\|
$$

$$
\left\|v^{-}\right\| \leq c\left\|u^{-}\right\|
$$

Injecting this into (3.31), we deduce that

which yields, owing to Gronwall's lemma,

$$
\frac{d}{d t}\left\|u^{-}\right\|^{2} \leq c\left\|u^{-}\right\|^{2}
$$

$$
\left\|u^{-}(t)\right\| \leq e^{c t}\left\|u^{-}(0)\right\|, t \geq 0
$$

whence, since $u^{-}(0)=0, u \geq 0$ a.e. $(x, t)$. This, together with (3.32), yields that $v \geq 0$ a.e. $(x, t)$. Consequently, $(u, v)$ is a strong solution to (3.1)-(3.4), which finishes the proof.

Remark 3.5. Proceeding exactly as in [6], we can prove that

$$
\|u(t)\|_{L^{\infty}(\Omega)} \leq\left\|u_{0}\right\|_{L^{\infty}(\Omega)}+(J+\kappa) t, t \geq 0 .
$$

Furthermore, it follows from (3.2) that 


$$
-\beta \Delta v+F v \leq F L+\kappa
$$

Multiplying (3.34) by $v^{m+1}, m \in \mathbb{N}$, we have

$$
\beta(m+1) \int_{\Omega} v^{m}|\nabla v|^{2} d x+F\|v\|_{L^{m+2}(\Omega)}^{m+2} \leq(F L+\kappa) \int_{\Omega} v^{m+1} d x,
$$

which yields

$$
F\|v\|_{L^{m+2}(\Omega)}^{m+2} \leq(F L+\kappa) \operatorname{Vol}(\Omega)^{\frac{1}{m+2}}\|v\|_{L^{m+2}(\Omega)}^{m+1},
$$

whence

$$
\|v\|_{L^{m+2}(\Omega)} \leq \frac{F L+\kappa}{F} \operatorname{Vol}(\Omega)^{\frac{1}{m+2}} .
$$

Passing to the limit $m \rightarrow+\infty$ in (3.35), we finally obtain (see, e.g., [3])

$$
\|v(t)\|_{L^{\infty}(\Omega)} \leq \frac{F L+\kappa}{F}, t \geq 0,
$$

meaning that the capillary lactate concentration is again uniformly (with respect to time) bounded. Also note that (2.10) still holds.

Remark 3.6. (i) As mentioned in the introduction (for the case $\varepsilon>0$, but the situation is the same here), the existence of solutions for negative initial data is a challenging issue. However, we can prove the following partial result (see also [6] for the case $\varepsilon>0$ ). Let $\delta_{1}$ and $\delta_{2}$ be two positive constants such that $k-\delta_{1}>0$ and $k^{\prime}-\delta_{2}>0$ and assume that $u_{0} \geq-\delta_{1}$ a.e. $x$. We then consider the following modified initial and boundary value problem:

$$
\begin{gathered}
\frac{\partial u}{\partial t}-\alpha \Delta u+\kappa\left(\frac{u}{k-\delta_{1}+\left|u+\delta_{1}\right|}-\frac{v}{k^{\prime}-\delta_{2}+\left|v+\delta_{2}\right|}\right)=J, \\
-\beta \Delta v+F v+\kappa\left(\frac{v}{k^{\prime}-\delta_{2}+\left|v+\delta_{2}\right|}-\frac{u}{k-\delta_{1}+\left|u+\delta_{1}\right|}\right)=F L, \\
\frac{\partial u}{\partial \nu}=\frac{\partial v}{\partial \nu}=0 \text { on } \Gamma, \\
\left.u\right|_{t=0}=u_{0} .
\end{gathered}
$$

The existence and uniqueness of the solution to (3.36)-(3.39) can be proved by arguing as above. Next, we set $\tilde{u}=u+\delta_{1}$ and $\tilde{v}=v+\delta_{2}$. These functions are solutions to

$$
\frac{\partial \tilde{u}}{\partial t}-\alpha \Delta \tilde{u}+\kappa\left(\frac{\tilde{u}}{k-\delta_{1}+|\tilde{u}|}-\frac{\tilde{v}}{k^{\prime}-\delta_{2}+|\tilde{v}|}\right)=\tilde{J}
$$




$$
\begin{gathered}
-\beta \Delta \tilde{v}+F \tilde{v}+\kappa\left(\frac{\tilde{v}}{k^{\prime}-\delta_{2}+|\tilde{v}|}-\frac{\tilde{u}}{k-\delta_{1}+|\tilde{u}|}\right)=\tilde{F} \\
\frac{\partial \tilde{u}}{\partial \nu}=\frac{\partial \tilde{v}}{\partial \nu}=0 \text { on } \Gamma \\
\left.\tilde{u}\right|_{t=0}=u_{0}+\delta_{1},
\end{gathered}
$$

where

and

$$
\tilde{J}=J+\kappa\left(\frac{\delta_{1}}{k-\delta_{1}+|\tilde{u}|}-\frac{\delta_{2}}{k^{\prime}-\delta_{2}+|\tilde{v}|}\right)
$$

$$
\tilde{F}=F\left(L+\delta_{2}\right)-\kappa\left(\frac{\delta_{1}}{k-\delta_{1}+|\tilde{u}|}-\frac{\delta_{2}}{k^{\prime}-\delta_{2}+|\tilde{v}|}\right) .
$$

Choosing $\delta_{1}$ and $\delta_{2}$ such that $\tilde{J} \geq 0$ and $\tilde{F} \geq 0$ (in particular, these hold when $\delta_{1}$ and $\delta_{2}$ are small enough) and noting that $\tilde{u}(0) \geq 0$ a.e. $x$, we can prove, as in the proof of Theorem 3.4 , that $\tilde{u}(x, t) \geq 0$ and $\tilde{v}(x, t) \geq 0$ a.e. $(x, t)$, so that $(u, v)$ is solution to (3.1)-(3.4), with

$$
u(x, t) \geq-\delta_{1} \text { and } v(x, t) \geq-\delta_{2} \text { a.e. }(x, t) .
$$

(ii) Similarly, we can prove that, if $\delta_{1}$ and $\delta_{2}$ are positive and small enough, with $u_{0} \geq \delta_{1}$ a.e. $x$, then

$$
u(x, t) \geq \delta_{1} \text { and } v(x, t) \geq \delta_{2} \text { a.e. }(x, t) .
$$

It follows from the above that we can actually define the Lipschitz continuous (for the $L^{2}$ and $H^{1}$-norms) mapping

$$
\mathcal{F}: H^{1}(\Omega) \rightarrow H^{1}(\Omega), w \mapsto z=\mathcal{F}(w),
$$

where $z$ is the unique solution to the following elliptic problem:

$$
a(z, \phi)+\left(\left(\varphi_{k^{\prime}}(z), \phi\right)\right)=\left(\left(F L+\varphi_{k}(w), \phi\right)\right), \forall \phi \in H^{1}(\Omega) .
$$

We then have the

Proposition 3.7. The mapping $\mathcal{F}$ is differentiable with respect to the $L^{2}$ and $H^{1}$-norms. Proof. Let $w_{0}$ and $w$ belong to $H^{1}(\Omega)$ and set $z_{0}=\mathcal{F}\left(w_{0}\right)$ and $z=\mathcal{F}(w)$. We then have

$$
a\left(z-z_{0}, \phi\right)+\left(\left(\varphi_{k^{\prime}}(z)-\varphi_{k^{\prime}}\left(z_{0}\right), \phi\right)\right)=\left(\left(\varphi_{k}(w)-\varphi_{k}\left(w_{0}\right), \phi\right)\right), \forall \phi \in H^{1}(\Omega) .
$$

Taking $\phi=z-z_{0}$ and reacalling tht $\varphi_{k^{\prime}}$ is monotone increasing, this yields

$$
\left\|z-z_{0}\right\|_{H^{1}(\Omega)} \leq c\left\|\varphi_{k}(w)-\varphi_{k}\left(w_{0}\right)\right\|
$$


whence

$$
\left\|z-z_{0}\right\|_{H^{1}(\Omega)} \leq c\left\|w-w_{0}\right\| .
$$

Let then $Z \in H^{1}(\Omega)$ be the solution to the linear elliptic problem (recall that $\varphi_{k}^{\prime}$ is nonnegative)

$$
a(Z, \phi)+\left(\left(\varphi_{k^{\prime}}^{\prime}\left(z_{0}\right) Z, \phi\right)\right)=\left(\left(\varphi_{k}^{\prime}\left(w_{0}\right)\left(w-w_{0}\right), \phi\right)\right), \forall \phi \in H^{1}(\Omega) .
$$

Setting $h=w-w_{0}$, we can see that

$$
\begin{aligned}
& a\left(z-z_{0}-Z, \phi\right)+\left(\left(\varphi_{k^{\prime}}(z)-\varphi_{k^{\prime}}\left(z_{0}\right)-\varphi_{k^{\prime}}^{\prime}\left(z_{0}\right) Z, \phi\right)\right) \\
& \quad=\left(\left(\varphi_{k}(w)-\varphi_{k}\left(w_{0}\right)-\varphi_{k}^{\prime}\left(w_{0}\right) h, \phi\right)\right), \forall \phi \in H^{1}(\Omega) .
\end{aligned}
$$

Writing

$$
\varphi_{k^{\prime}}(z)-\varphi_{k^{\prime}}\left(z_{0}\right)-\varphi_{k^{\prime}}^{\prime}\left(z_{0}\right) Z=\varphi_{k^{\prime}}^{\prime}\left(z_{0}\right)\left(z-z_{0}-Z\right)+o\left(\left\|z-z_{0}\right\|\right)
$$

and

$$
\varphi_{k}(w)-\varphi_{k}\left(w_{0}\right)-\varphi_{k}^{\prime}\left(w_{0}\right) h=o(\|h\|),
$$

we obtain, taking $\phi=z-z_{0}-Z$ and employing (3.46) (also recall that $\varphi_{k^{\prime}}^{\prime} \geq 0$ ),

$$
a\left(z-z_{0}-Z, z-z_{0}-Z\right) \leq\left|\left(\left(o(\|h\|), z-z_{0}-Z\right)\right)\right|
$$

whence

$$
\left\|z-z_{0}-Z\right\|_{H^{1}(\Omega)}=o(\|h\|) .
$$

This yields that $\mathcal{F}$ is differentiable at $w_{0}$, with $\mathcal{F}^{\prime}\left(w_{0}\right) \cdot h=Z, \mathcal{F}^{\prime}$ denoting the differential of $\mathcal{F}$.

We deduce from Proposition 3.7 the

Corollary 3.8. Let $(u, v)$ be the solution to (3.1)-(3.4) given in Theorem 3.4. Then, $\forall T>0$,

$$
\frac{\partial v}{\partial t} \in L^{\infty}\left(0, T ; H^{1}(\Omega)\right)
$$

and

$$
\left\|\frac{\partial v}{\partial t}\right\|_{H^{1}(\Omega)} \leq c\left\|\frac{\partial u}{\partial t}\right\| \text { a.e. } t \geq 0
$$


Proof. It suffices to note that $v=\mathcal{F}(u)$, whence, owing to Proposition 3.7,

$$
\frac{\partial v}{\partial t}=\mathcal{F}^{\prime}(u) \cdot \frac{\partial u}{\partial t}
$$

Indeed, we can note that $u \in H^{1}\left(0, T ; H^{1}(\Omega)\right), \forall T>0$. Furthermore, we have

$$
a\left(\frac{\partial v}{\partial t}, \phi\right)+\left(\left(\varphi_{k^{\prime}}^{\prime}(v) \frac{\partial v}{\partial t}, \phi\right)\right)=\left(\left(\varphi_{k}^{\prime}(u) \frac{\partial u}{\partial t}, \phi\right)\right), \forall \phi \in H^{1}(\Omega),
$$

and (3.49) follows, taking $\phi=\frac{\partial v}{\partial t}$ (also recall that $\frac{\partial u}{\partial t} \in L^{\infty}\left(0, T ; L^{2}(\Omega)\right), \forall T>0$ ).

Remark 3.9. It follows from standard elliptic regularity results applied to (3.51) (see, e.g., [1] and [2]) that, $\forall T>0, \frac{\partial v}{\partial t} \in L^{\infty}\left(0, T ; H^{2}(\Omega)\right) \cap L^{2}\left(0, T ; H^{3}(\Omega)\right)$, with

and

$$
\left\|\frac{\partial v}{\partial t}\right\|_{H^{2}(\Omega)} \leq c\left\|\frac{\partial u}{\partial t}\right\| \text { a.e. } t \geq 0
$$

$$
\left\|\frac{\partial v}{\partial t}\right\|_{L^{2}\left(0, T ; H^{3}(\Omega)\right)} \leq Q\left(T,\left\|u_{0}\right\|_{H^{1}(\Omega)}\right)\left\|\frac{\partial u}{\partial t}\right\|_{L^{2}\left(0, T ; H^{1}(\Omega)\right)} .
$$

\section{Convergence to the limit problem}

All constants $c$ and $c^{\prime}$ and functions $Q$ in this section are independent of $\varepsilon$.

Let $\left(u^{\varepsilon}, v^{\varepsilon}\right)$ and $\left(u^{0}, v^{0}\right)$ be the unique strong solutions to the initial and limit problems, respectively, as given in Theorems 2.1 and 3.4, where $v_{0}=v^{0}(0)$, i.e.,

$$
\begin{gathered}
\frac{\partial u^{\varepsilon}}{\partial t}-\alpha \Delta u^{\varepsilon}+\kappa\left(\frac{k^{\prime}}{k^{\prime}+v^{\varepsilon}}-\frac{k}{k+u^{\varepsilon}}\right)=J, \\
\varepsilon \frac{\partial v^{\varepsilon}}{\partial t}-\beta \Delta v^{\varepsilon}+F v^{\varepsilon}+\kappa\left(\frac{k}{k+u^{\varepsilon}}-\frac{k^{\prime}}{k^{\prime}+v^{\varepsilon}}\right)=F L, \\
\frac{\partial u^{\varepsilon}}{\partial \nu}=\frac{\partial v^{\varepsilon}}{\partial \nu}=0 \text { on } \Gamma, \\
\left.u^{\varepsilon}\right|_{t=0}=u_{0},\left.v^{\varepsilon}\right|_{t=0}=v^{0}(0),
\end{gathered}
$$

and

$$
\begin{gathered}
\frac{\partial u^{0}}{\partial t}-\alpha \Delta u^{0}+\kappa\left(\frac{k^{\prime}}{k^{\prime}+v^{0}}-\frac{k}{k+u^{0}}\right)=J, \\
-\beta \Delta v^{0}+F v^{0}+\kappa\left(\frac{k}{k+u^{0}}-\frac{k^{\prime}}{k^{\prime}+v^{0}}\right)=F L,
\end{gathered}
$$




$$
\begin{gathered}
\frac{\partial u^{0}}{\partial \nu}=\frac{\partial v^{0}}{\partial \nu}=0 \text { on } \Gamma, \\
\left.u^{0}\right|_{t=0}=u_{0} .
\end{gathered}
$$

We have the

Theorem 4.1. The following error estimates hold, $\forall T>0$ :

$$
\left\|u^{\varepsilon}(t)-u^{0}(t)\right\|_{H^{1}(\Omega)} \leq Q\left(T,\left\|u_{0}\right\|_{H^{1}(\Omega)}\right) \varepsilon,\left\|v^{\varepsilon}(t)-v^{0}(t)\right\|_{H^{1}(\Omega)} \leq Q\left(T,\left\|u_{0}\right\|_{H^{1}(\Omega)}\right) \sqrt{\varepsilon}
$$

$t \in[0, T]$, and

$$
\left\|u^{\varepsilon}-u^{0}\right\|_{L^{2}\left(0, T ; H^{2}(\Omega)\right)} \leq Q\left(T,\left\|u_{0}\right\|_{H^{1}(\Omega)}\right) \varepsilon,\left\|v^{\varepsilon}-v^{0}\right\|_{L^{2}\left(0, T ; H^{2}(\Omega)\right)} \leq Q\left(T,\left\|u_{0}\right\|_{H^{1}(\Omega)}\right) \varepsilon .
$$

Proof. We have, setting $u=u^{\varepsilon}-u^{0}$ and $v=v^{\varepsilon}-v^{0}$,

$$
\begin{gathered}
\frac{\partial u}{\partial t}-\alpha \Delta u+\varphi_{k}\left(u^{\varepsilon}\right)-\varphi_{k}\left(u^{0}\right)-\varphi_{k^{\prime}}\left(v^{\varepsilon}\right)+\varphi_{k^{\prime}}\left(v^{0}\right)=0 \\
\varepsilon \frac{\partial v}{\partial t}-\beta \Delta v+F v+\varphi_{k^{\prime}}\left(v^{\varepsilon}\right)-\varphi_{k^{\prime}}\left(v^{0}\right)-\varphi_{k}\left(u^{\varepsilon}\right)+\varphi_{k}\left(u^{0}\right)=-\varepsilon \frac{\partial v^{0}}{\partial t}, \varepsilon>0, \\
\frac{\partial u}{\partial \nu}=\frac{\partial v}{\partial \nu}=0 \text { on } \Gamma, \\
\left.u\right|_{t=0}=0,\left.v\right|_{t=0}=0 .
\end{gathered}
$$

Multiplying (4.9) by $u$, we obtain, recalling that $\varphi_{k}$ is monotone increasing,

$$
\frac{d}{d t}\|u\|^{2}+\alpha\|\nabla u\|^{2} \leq c\|u\|\|v\| .
$$

Multiplying then (4.10) by $v$, we find, similarly,

$$
\varepsilon \frac{d}{d t}\|v\|^{2}+c\|v\|_{H^{1}(\Omega)}^{2} \leq c^{\prime}\left(\|u\|\|v\|+\varepsilon^{2}\left\|\frac{\partial v^{0}}{\partial t}\right\|^{2}\right), c>0 .
$$

Combining (4.13) and (4.14), we have, owing to (3.49),

$$
\frac{d}{d t}\left(\|u\|^{2}+\varepsilon\|v\|^{2}\right)+c\left(\|u\|_{H^{1}(\Omega)}^{2}+\|v\|_{H^{1}(\Omega)}^{2}\right) \leq c^{\prime}\left(\|u\|^{2}+\varepsilon\|v\|^{2}+\varepsilon^{2}\left\|\frac{\partial u^{\varepsilon}}{\partial t}\right\|^{2}\right), c>0,
$$

from which it follows, owing to Gronwall's lemma,

$$
\|u(t)\|^{2}+\varepsilon\|v(t)\|^{2}+c \int_{0}^{T}\left(\|u(s)\|_{H^{1}(\Omega)}^{2}+\|v(s)\|_{H^{1}(\Omega)}^{2}\right) d s
$$




$$
\leq Q(T) \varepsilon^{2}\left\|\frac{\partial u^{\varepsilon}}{\partial t}\right\|_{L^{2}\left(0, T ; L^{2}(\Omega)\right)}^{2}, c>0, t \in[0, T] .
$$

Multiplying now (4.1) by $\frac{\partial u^{\varepsilon}}{\partial t}$, we obtain

whence

$$
\frac{d}{d t}\left\|\nabla u^{\varepsilon}\right\|^{2}+c\left\|\frac{\partial u^{\varepsilon}}{\partial t}\right\|^{2} \leq c^{\prime}, c>0
$$

$$
\left\|\frac{\partial u^{\varepsilon}}{\partial t}\right\|_{L^{2}\left(0, T ; L^{2}(\Omega)\right)}^{2} \leq Q\left(T,\left\|u_{0}\right\|_{H^{1}(\Omega)}\right)
$$

We finally deduce from (4.16)-(4.17) that

$$
\begin{aligned}
\|u(t)\|^{2}+ & \varepsilon\|v(t)\|^{2}+c \int_{0}^{T}\left(\|u(s)\|_{H^{1}(\Omega)}^{2}+\|v(s)\|_{H^{1}(\Omega)}^{2}\right) d s \\
& \leq Q\left(T,\left\|u_{0}\right\|_{H^{1}(\Omega)}\right) \varepsilon^{2}, c>0, t \in[0, T] .
\end{aligned}
$$

Multiplying next (4.9) by $-\Delta u$ and (4.10) by $-\Delta v$, we find, recalling that $\varphi_{k}$ and $\varphi_{k^{\prime}}$ are Lipschitz continuous,

and

$$
\frac{d}{d t}\|\nabla u\|^{2}+c\|\Delta u\|^{2} \leq c^{\prime}\left(\|u\|^{2}+\|v\|^{2}\right), c>0,
$$

$$
\varepsilon \frac{d}{d t}\|\nabla v\|^{2}+c\|\Delta v\|^{2} \leq c^{\prime}\left(\|u\|^{2}+\|v\|^{2}+\varepsilon^{2}\left\|\frac{\partial v^{0}}{\partial t}\right\|^{2}\right), c>0,
$$

respectively.

Summing these two inequalities, integrating over $[0, T]$ and proceeding as above, we have, adding the resulting inequality to (4.18),

$$
\begin{gathered}
\|u(t)\|_{H^{1}(\Omega)}^{2}+\varepsilon\|v(t)\|_{H^{1}(\Omega)}^{2}+c \int_{0}^{T}\left(\|u(s)\|_{H^{2}(\Omega)}^{2}+\|v(s)\|_{H^{2}(\Omega)}^{2}\right) d s \\
\leq c^{\prime}\left(\int_{0}^{T}\left(\|u(s)\|^{2}+\|v(s)\|^{2}\right) d s+Q\left(T,\left\|u_{0}\right\|_{H^{1}(\Omega)}\right) \varepsilon^{2}\right), c>0 .
\end{gathered}
$$

This yields, employing (4.18) to estimate the right-hand side,

$$
\begin{aligned}
\|u(t)\|_{H^{1}(\Omega)}^{2}+ & \varepsilon\|v(t)\|_{H^{1}(\Omega)}^{2}+c \int_{0}^{T}\left(\|u(s)\|_{H^{2}(\Omega)}^{2}+\|v(s)\|_{H^{2}(\Omega)}^{2}\right) d s \\
& \leq Q\left(T,\left\|u_{0}\right\|_{H^{1}(\Omega)}\right) \varepsilon^{2}, c>0, t \in[0, T],
\end{aligned}
$$

which finishes the proof.

Remark 4.2. We have similar error estimates if we assume that $\left\|u^{\varepsilon}(0)-u^{0}(0)\right\|_{H^{1}(\Omega)} \leq c \varepsilon$ and $\left\|v^{\varepsilon}(0)-v^{0}(0)\right\|_{H^{1}(\Omega)} \leq c \sqrt{\varepsilon}$. 


\section{A StABILITy Result}

As in [6], (3.1)-(3.2) possesses a unique spatially homogeneous equilibrium $(\bar{u}, \bar{v})$ given by

and

$$
\bar{v}=L+\frac{J}{F}>0
$$

$$
\bar{u}=\frac{k\left(\frac{J}{\kappa}+\frac{\bar{v}}{k^{\prime}+\bar{v}}\right)}{1-\left(\frac{J}{\kappa}+\frac{\bar{v}}{k^{\prime}+\bar{v}}\right)} .
$$

Note that $\bar{u}$ is not necessarily positive. We thus assume in what follows that

$$
\bar{u}>0 .
$$

The linearized (around $(\bar{u}, \bar{v})$ ) system reads

$$
\begin{gathered}
\frac{\partial U}{\partial t}-\alpha \Delta U+\kappa\left(\frac{k}{(k+\bar{u})^{2}} U-\frac{k^{\prime}}{\left(k^{\prime}+\bar{v}\right)^{2}} V\right)=0 \\
-\beta \Delta V+F V+\kappa\left(\frac{k^{\prime}}{\left(k^{\prime}+\bar{v}\right)^{2}} V-\frac{k}{(k+\bar{u})^{2}} U\right)=0 \\
\frac{\partial U}{\partial \nu}=\frac{\partial V}{\partial \nu}=0 \text { on } \Gamma \\
\left.U\right|_{t=0}=U_{0} .
\end{gathered}
$$

It is not difficult here to prove the existence, uniqueness and regularity of the solution to (5.1)-(5.4), assuming that $U_{0}$ is regular enough. Furthermore, proceeding as above, we can prove that, if $U_{0} \geq 0$ a.e. $x$, then $U(x, t) \geq 0$ and $V(x, t) \geq 0$ a.e. $(x, t)$.

Multiplying now $(5.1)$ by $\frac{k}{(k+\bar{u})^{2}} U$ and $(5.2)$ by $\frac{k^{\prime}}{\left(k^{\prime}+\bar{v}\right)^{2}} V$, we obtain, summing the two resulting equalities,

$$
\begin{gathered}
\frac{1}{2} \frac{k}{(k+\bar{u})^{2}} \frac{d}{d t}\|U\|^{2}+\frac{\alpha k}{(k+\bar{u})^{2}}\|\nabla U\|^{2}+\frac{\beta k^{\prime}}{\left(k^{\prime}+\bar{v}\right)^{2}}\|\nabla V\|^{2}+\frac{F k^{\prime}}{\left(k^{\prime}+\bar{v}\right)^{2}}\|V\|^{2} \\
+\int_{\Omega}\left(\frac{k}{(k+\bar{u})^{2}} U-\frac{k^{\prime}}{\left(k^{\prime}+\bar{v}\right)^{2}} V\right)^{2} d x=0 .
\end{gathered}
$$

It follows from (5.5) that

whence

$$
\frac{d}{d t}\|U\|^{2} \leq 0
$$

$$
\|U(t)\| \leq\left\|U_{0}\right\|, t \geq 0 .
$$


Multiplying next (5.2) by $V$, we easily find

so that

$$
\|V\| \leq c\|U\|
$$

$$
\|V(t)\| \leq c\left\|U_{0}\right\|, t \geq 0
$$

We deduce from $(5.6)-(5.7)$ that $(\bar{u}, \bar{v})$ is linearly stable with respect to the $L^{2}$-norm. We can also prove the linear stability with respect to the $H^{1}$-norm, proceeding in a similar way.

Now, an important question is whether we also have a linear exponential stability as in [6] for the case $\varepsilon>0$ (see also [5], [8], [9] and [10] for the ODE's model (1.1)-(1.2)). Indeed, as mentioned in the introduction, a therapeutic perspective of such a result is to have the (spatially homogeneous) steady state outside the viability domain, where cell necrosis occurs (see [8]).

We have, in this direction, the

Theorem 5.1. The stationary solution $(\bar{u}, \bar{v})$ is linearly exponentially stable, in the sense that all eigenvalues $s \in \mathbb{C}$ associated with the linear system (5.1)-(5.2) satisfy $\mathcal{R} e(s) \leq-\xi$, for a given $\xi>0, \mathcal{R} e$ denoting the real part.

Proof. We first note that it follows from (5.2) that

$$
V=k_{1}\left(-\beta \Delta+\left(F+k_{2}\right) I\right)^{-1} U
$$

where $k_{1}=\frac{\kappa k}{(k+\bar{u})^{2}}$ and $k_{2}=\frac{\kappa k^{\prime}}{\left(k^{\prime}+\bar{v}\right)^{2}}$. Injecting this into (5.1), we obtain

$$
\frac{\partial U}{\partial t}-\alpha \Delta U+k_{1} U-k_{1} k_{2}\left(-\beta \Delta+\left(F+k_{2}\right) I\right)^{-1} U=0 .
$$

We then look for solutions of the form

$$
U(x, t)=\hat{U}(x) e^{s t}
$$

for $s \in \mathbb{C}, s=\zeta+i \eta$. Injecting this into (5.9), we find

$$
-\alpha \Delta \hat{U}+\left(s+k_{1}\right) \hat{U}-k_{1} k_{2}\left(-\beta \Delta+\left(F+k_{2}\right) I\right)^{-1} \hat{U}=0
$$

where

$$
\frac{\partial \hat{U}}{\partial \nu}=0 \text { on } \Gamma
$$

This yields

$$
\alpha \beta \Delta^{2} \hat{U}-\left(\beta s+\alpha F+\beta k_{1}+\alpha k_{2}\right) \Delta \hat{U}+\left(\left(F+k_{2}\right) s+k_{1} F\right) \hat{U}=0,
$$

where, owing to (5.10) and (5.11), 


$$
\frac{\partial \hat{U}}{\partial \nu}=\frac{\partial \Delta \hat{U}}{\partial \nu}=0 \text { on } \Gamma
$$

Multiplying (5.12) by the conjugate of $\hat{U}$, integrating over $\Omega$ and by parts and taking the real part, we have

$$
\alpha \beta\||\Delta \hat{U}|\|^{2}+\left(\beta \zeta+\alpha F+\beta k_{1}+\alpha k_{2}\right)\||\nabla \hat{U}|\|^{2}+\left(\left(F+k_{2}\right) \zeta+k_{1} F\right)\||\hat{U}|\|^{2}=0 .
$$

Therefore, when $\zeta \geq 0$, then, necessarily, $\hat{U} \equiv 0$. Furthermore, (5.14) can have nontrivial solutions only when

$$
\beta \zeta+\alpha F+\beta k_{1}+\alpha k_{2} \leq 0
$$

or

$$
\left(F+k_{2}\right) \zeta+k_{1} F \leq 0
$$

Therefore, necessarily,

$$
\zeta \leq \max \left(-\frac{\alpha F+\beta k_{1}+\alpha k_{2}}{\beta},-\frac{k_{1} F}{F+k_{2}}\right)<0,
$$

which finishes the proof.

\section{REFERENCES}

[1] S. Agmon, A. Douglis and L. Nirenberg, Estimates near the boundary for solutions of elliptic partial differential equations, I, Commun. Pure Appl. Math. 12 (1959), 623-727.

[2] S. Agmon, A. Douglis and L. Nirenberg, Estimates near the boundary for solutions of elliptic partial differential equations, II, Commun. Pure Appl. Math. 17 (1964), 35-92.

[3] N.D. Alikakos, $L^{p}$ bounds of solutions to reaction-diffusion equations, Commun. Partial Differ. Eqns. 4 (1979), 827-868.

[4] A. Aubert and R. Costalat, Interaction between astrocytes and neurons studied using a mathematical model of compartmentalized energy metabolism, J. Cereb. Blood Flow Metab. 25 (2005), 1476-1490.

[5] R. Costalat, J.-P. Françoise, C. Menuel, M. Lahutte, J.-N. Vallée, G. de Marco, J. Chiras and R. Guillevin, Mathematical modeling of metabolism and hemodynamics, Acta Biotheor. 60 (2012), 99-107.

[6] R. Guillevin, A. Miranville and A. Perrillat, On a reaction-diffusion system associated with brain lactate kinetics, submitted.

[7] Y. Hu and G.S. Wilson, A temporary local energy pool coupled to neuronal activity: fluctuations of extracellular lactate levels in rat brain monitored with rapid-response enzyme-based sensor, J. Neurochem. 69 (1997), 1484-1490.

[8] M. Lahutte-Auboin, Modélisation biomathématique du métabolisme énergétique cérébral : réduction de modèle et approche multi-échelle, application à l'aide à la décision pour la pathologie des gliomes, $\mathrm{PhD}$ thesis, Université Pierre et Marie Curie, 2015.

[9] M. Lahutte-Auboin, R. Costalat, J.-P. Françoise and R. Guillevin, Dip and buffering in a fast-slow system associated to brain lactate kinetics, Preprint, 2013. 
[10] M. Lahutte-Auboin, R. Guillevin, J.-P. Françoise, J.-N. Vallée and R. Costalat, On a minimal model for hemodynamics and metabolism of lactate: application to low grade glioma and therapeutic strategies, Acta Biotheor. 61 (2013), 79-89.

[11] J. Keener and J. Sneyd, Mathematical physiology, Second edition, Interdisciplinary Applied Mathematics, Vol. 8, Springer-Verlag, New York, 2009.

[12] A. Miranville, A singular reaction-diffusion equation associated with brain lactate kinetics, Math. Models Appl. Sci., to appear.

[13] R. Temam, Infinite-dimensional dynamical systems in mechanics and physics, Second edition, Applied Mathematical Sciences, Vol. 68, Springer-Verlag, New York, 1997.

[14] R. Temam, Navier-Stokes equations: theory and numerical analysis, AMS Chelsea Publishing, American Mathematical Society, Providence, Rhode Island, 2001.

Université De Poitiers

Laboratoire de Mathématiques et Applications

UMR CNRS 7348 - SP2MI

EQUIPE DACTIM-MIS

Boulevard Marie et Pierre Curie - Téléport 2

F-86962 Chasseneull Futuroscope Cedex, France

E-mail address: Alain.Miranville@math.univ-poitiers.fr 